\title{
COMPARACIÓN DE LAS PERCEPCIONES DE LOS ESTUDIANTES DE MAGISTERIO SOBRE LOS RIESGOS DE INTERNET PARA PERSONAS CON Y SIN DISCAPACIDAD INTELECTUAL Y DEL DESARROLLO
}

\author{
Marcos Gómez-Puerta \\ marcos.gomez@ua.es \\ Esther Chiner \\ Universidad de Alicante, España
}

Fecha de Recepción: 3 Abril 2018

Fecha de Admisión: 10 Abril 2018

\section{RESUMEN}

A pesar de que Internet tiene múltiples beneficios para la sociedad, también implica algunos riesgos que deben ser identificados. Estos riesgos se pueden percibir como mayores para algunos grupos más vulnerables como son los niños y las personas con discapacidad. No obstante, dichos riesgos no deben limitar el acceso a Internet, especialmente de las personas con discapacidad intelectual y del desarrollo (IDD). Por el contrario, se debería tener un mayor conocimiento sobre los peligros y cómo gestionarlos. El propósito de este estudio es explorar y comparar las percepciones que tienen los estudiantes de Magisterio sobre los riesgos del uso de Internet por personas con y sin IDD. Participó una muestra de conveniencia de 121 estudiantes de Magisterio de los cuales el $77 \%$ eran mujeres $(n=93)$ y la edad media de 21.79 años $(D T=4.23)$. Los participantes debían responder a un cuestionario que incluía algunas cuestiones sociodemográficas y 30 riesgos potenciales de Internet que se presentaron en una escala tipo Likert de cinco puntos ( $1=$ Totalmente en desacuerdo; 5 = Totalmente de acuerdo). Los estudiantes debían valorar cada ítem dos veces, una para cada uno de los colectivos estudiados (personas con y sin IDD). A pesar de que los participantes perciben un alto riesgo en el uso de Internet, se encontraron diferencias estadísticamente significativas entre los grupos. Los estudiantes de Magisterio perciben mayores riesgos en Internet para las personas con IDD que para el resto de la población en cuestiones como el ciberacoso, ser expuesto a contenido inapropiado, comunicarse con desconocidos, pasar demasiado tiempo en las redes sociales o gastar dinero online.

Palabras clave: internet; discapacidad intelectual y del desarrollo; riesgos; magisterio

\section{ABSTRACT}

Comparing student teachers' perceptions of the risks of the Internet for people with and without intellectual and developmental disabilities. 


\section{COMPARACIÓN DE LAS PERCEPCIONES DE LOS ESTUDIANTES DE MAGISTERIO SOBRE LOS RIESGOS DE INTERNET PARA PERSONAS CON Y SIN DISCAPACIDAD INTELECTUAL Y DEL DESARROLLO}

Although the Internet has multiple benefits for the society, it also involves some risks that must be identified. These risks may be perceived to be greater for some vulnerable groups such as children or people with disabilities. However, these inherent risks should not prevent people, and especially those with intellectual and developmental disabilities (IDD), from gaining online access. Instead, they should learn about the dangers and how to manage them. The purpose of this study is to explore the perceptions that student teachers have about risks of Internet use by people with and without IDD in order to compare them. A convenience sample of 121 student teachers was recruited into the study from which $77 \%$ were female students $(n=93)$ and the mean age was 21.79 years old $(S D=4.23)$. Participants had to respond to a questionnaire that included some demographic data and 30 potential Internet risks that were presented in a five-point Likert scale $(1=$ totally disagree; 5 = totally agree). Student teachers had to rate each risk twice, one for each of the groups investigated (people with IDD and without IDD). Although participants perceive Internet risks to be high for everyone, significant differences were found between groups. Student teachers perceive the Internet to be more risky for people with IDD than for the general population. Differences were particularly greater for questions related to cyberbullying, being exposed to inappropriate content, communicating with people not known to them or their families, spending too much time on social networking sites, and spending money online, among others.

Keywords: internet; intellectual and developmental disability; risks; teaching

\section{ANTECEDENTES}

La incorporación de las tecnologías de la información y de la comunicación (TIC) en todos los ámbitos de nuestra vida han supuesto un gran avance para la sociedad, contribuyendo de manera significativa a fomentar una mayor calidad de vida y mejores resultados en el ámbito social, personal y laboral de todas las personas (Palmer, Wehmeyer, Davies, \& Stock, 2012). El acceso a las TIC, y en especial a Internet, ha facilitado también la inclusión, no solo social sino también digital, de otros grupos que tradicionalmente han sido excluidos de participar de forma plena en la sociedad (Normand et al., 2016). Este es el caso de las personas con discapacidad intelectual y del desarroIlo (IDD) las cuales han encontrado en la Red otras formas de relacionarse y participar. Si bien la brecha digital para este colectivo todavía es patente (Caton \& Chapman, 2016), cada vez son más las personas con IDD que acceden a Internet y disfrutan de las mismas ventajas que el resto de la población (Chiner, Gómez-Puerta, \& Cardona-Moltó, 2017b; Gutiérrez \& Martorell, 2011).

El acceso a Internet ofrece múltiples beneficios tanto para la sociedad en general como para las personas con IDD. Concretamente, Internet ofrece a este colectivo mayores oportunidades de interacción social, participación, educación, trabajo y desarrollo de la identidad, entre otras (Chadwick, Fullwood, \& Wesson, 2013). Sin embargo, junto a estas ventajas, Internet puede también comportar una serie de riesgos que, en algunos casos, pueden ser percibidos como mayores para las personas con IDD. Ciertos prejuicios hacia estas personas en el mundo real (World Health Organization, 2011), pueden también trasladarse al mundo online (Chadwick \& Wesson, 2016). En muchas ocasiones, existe una sobreprotección hacia este grupo por la percepción generalizada, especialmente de sus cuidadores (e.g. familia, profesionales, profesores), de que pueden ser más vulnerables a los peligros de la sociedad y más concretamente del entorno online (e.g. perder dinero, recibir abusos, dar credibilidad a información falsa). En general, se entiende la discapacidad intelectual y del desarrollo como limitaciones significativas en el funcionamiento intelectual y en la conducta adaptativa de las personas, que se manifiestan en sus habilidades adaptativas conceptuales, sociales y prácticas, y que aparece antes de los 18 años (Schalock et al., 2010), y son precisamente estas características las que llevan a pensar que pueden ser más fácilmente víctimas de conductas inapropiadas en Internet. 
La percepción de un mayor riesgo de Internet para las personas con IDD puede tener una consecuencia directa en el uso que estas personas hacen de la Red, limitando sus oportunidades de acceder a ella y, por tanto, reduciendo sus posibilidades de disfrutar de los beneficios que ofrece. Teniendo en cuenta que las personas encargadas de la atención y cuidado de las personas con IDD son su principal apoyo en el uso de las tecnologías (Palmer et al., 2012), las percepciones que estos tengan sobre los riesgos de Internet y su capacidad para gestionarlos pueden determinar el grado en que las personas con IDD acceden a Internet (Seale, 2014). En muchos casos, los temores de los cuidadores y la necesidad de proteger a este colectivo puede llevar a limitaciones o restricciones en el uso de Internet y a un mayor control de las actividades que desarrollan en la Red (LöfgrenMårtenson, 2008), perpetuando, de esta forma, la brecha digital.

La solución puede pasar por conocer en profundidad las percepciones que tiene la sociedad, y en particular, las personas cercanas sobre el uso de Internet por parte de las personas con IDD y, en particular, sobre los riesgos que ellos consideran que están presentes cuando acceden a la Red, así como su capacidad de afrontarlos y gestionarlos de forma constructiva. En este sentido, Chadwick, Quinn y Fullwood (2017) señalan en un estudio sobre las percepciones que tienen las personas sin IDD sobre los beneficios y riesgos de Internet tanto para ellos mismos como para las personas con IDD, que la población general tiene algunos conceptos erróneos sobre el acceso y uso de Internet por personas con IDD. De acuerdo con este estudio, las personas sin IDD perciben que los beneficios, y especialmente los riesgos, son mayores para las personas con IDD que para la población general. En un estudio similar también se observó que tanto los familiares como los profesionales al cargo de personas con IDD consideran que Internet comporta riesgos para este colectivo (Chiner, Gómez-Puerta, \& Cardona-Moltó, 2017a).

A pesar de la importancia y la necesidad de identificar los riesgos y de conocer las percepciones de determinados grupos acerca del uso de Internet por personas con IDD la investigación sobre este tema es escasa. Existen estudios que tratan de conocer la prevalencia del ciberacoso y otras conductas online en la población general como, por ejemplo, en niños y adolescentes (Jones, Mitchell, \& Finkelhor, 2012; e.g. Livingstone \& Haddon, 2009), pero poco se sabe sobre este tema en colectivos más específicos como son las personas con IDD. Estudios como los de Didden et al. (2009), Jenaro et al. (2018) y Wells y Mitchell (2014) tratan de analizar estos aspectos, Ilegando a la conclusión de que las personas con IDD tienen más probabilidades de sufrir ciberacoso y que esta situación se puede dar con mayor frecuencia en los entornos educativos.

\section{OBJETIVOS}

Tomando como referencia las investigaciones previas resulta necesario seguir profundizando en estos aspectos, especialmente en el ámbito educativo, donde parece que se producen con mayor frecuencia estas conductas. Por ello, siguiendo la línea del estudio realizado por Chadwick et al. (2017) en el que se analizaban las percepciones de la población general sobre los riesgos de Internet para personas con y sin IDD, y considerando el papel relevante que tienen los maestros en el fomento del uso de las TIC y de Internet, hemos querido centrarnos en conocer las percepciones que tienen los docentes sobre estos temas, no solo respecto a la población general, sino también respecto a los alumnos con IDD con el objeto de conocer cuáles son sus principales temores y en qué medida difieren según la población a la que nos refiramos.

Por todo lo anterior, el propósito de este estudio es explorar y comparar las percepciones de los estudiantes de Magisterio acerca de los riesgos que tiene el uso de Internet para personas con y sin discapacidad intelectual y del desarrollo (IDD). En concreto nos plateamos las siguientes preguntas de investigación: 
¿En qué medida perciben los estudiantes de Magisterio que el uso de Internet conlleva riesgos para las personas con y sin IDD?

¿Existen diferencias estadísticamente significativas en las percepciones que tienen los estudiantes de Magisterio de los riesgos online para cada una de las poblaciones estudiadas (personas con y $\sin (D D)$ ?

\section{PARTICIPANTES}

En el estudio participó una muestra de conveniencia de 121 estudiantes de Magisterio de los cuales el $77 \%(n=93)$ eran mujeres y el $23 \%(n=28)$ eran hombres. Sus edades comprendían entre los 17 y los 42 años, con una edad media de $21.79(D T=4.23)$. La mayoría de los estudiantes apenas tenía contacto con personas con IDD (76\%), un $8 \%$ lo tenía mensualmente y un $16 \%$ regularmente.

\section{MÉTODO E INSTRUMENTOS}

Se llevó a cabo un estudio de corte cuantitativo, no experimental descriptivo. Con el objeto de conocer las percepciones que tienen los estudiantes de Magisterio sobre el uso de Internet, se les administró la versión online de un cuestionario diseñado para este estudio a partir de la adaptación de los trabajos previos de Livingstone, Haddon, Görzig y Ólfasson (2011) y de Chadwick et al. (2017). El instrumento incluía información sociodemográfica y un listado 30 ítems relativos a diferentes tipos de riesgos que se pueden dar cuando se accede a Internet. Los participantes debían responder al cuestionario a través de una escala tipo Likert de cinco puntos en la que 1 era totalmente en desacuerdo y 5 totalmente de acuerdo. Además, debían valorar cada uno de los ítems dos veces, según se refiriera a personas con IDD o personas sin IDD. El instrumento muestra una buena consistencia interna con un valor alpha de .96 para cada una de las escalas (personas con IDD y personas $\sin$ IDD).

Se solicitó la colaboración de los estudiantes de Magisterio para que respondieran a la versión online del cuestionario a través de cualquier dispositivo (e.g. móvil, ordenador, tableta). El tiempo de respuesta no superaba los 10 minutos. Previo a la cumplimentación del cuestionario se solicitó el consentimiento informado de los participantes y se contó con la aprobación del Comité de ética de la Universidad de Alicante. A los participantes se les garantizó el anonimato de sus respuestas. El análisis de los datos se llevó a cabo a través estadísticos descriptivos (porcentajes, media y desviación típica) y para la comparación de las valoraciones de los estudiantes sobre los riesgos de Internet entre personas con y $\sin$ IDD se realizaron diversas pruebas $t$ para muestras relacionadas.

\section{RESULTADOS}

Percepciones de los riesgos del uso de Internet para personas con y sin discapacidad intelectual y del desarrollo

Los resultados indican que los estudiantes de Magisterio perciben Internet como un entorno en el que existen ciertos riesgos tanto para las personas con IDD como para la población general. De hecho, la percepción de los riesgos tanto para un grupo para como el otro fueron valorados, en su mayoría, con puntuaciones superiores a 4 (en una escala del 1 al 5) con una puntuación media de $4.39(D T=0.55)$ para las personas con IDD y de $4.14(D T=0.63)$ en el caso de las personas sin IDD. Concretamente, tal como se observa en la Tabla 1, los riegos con valoraciones más altas para las personas con IDD fueron: ser acosado $(M=4.64, D T=0.61)$, tener contacto con personas desconocidas $(M=4.55, D T=0.75)$, aportar demasiada información personal $(M=4.58, D T=0.64)$, ser amenazado $(M=4.55, D T=0.70)$ y tener dificultad para discriminar la veracidad de la informa- 
ción $(M=4.56, D T=0.66)$. Por otro lado, de acuerdo con los estudiantes de Magisterio, las mayores amenazas de Internet para las personas sin IDD son ser acosado online $(M=4.24, D T=0.85)$, ser expuesto a material que fomente la conducta antisocial y la intolerancia $(M=4.25, D T=0.86)$, el uso indebido de sus datos personales $(M=4.30, D T=0.81)$, descargar involuntariamente spyware o malware en su ordenador $(M=4.26, D T=0.89)$ y difundir fotos 0 información personal de otras personas $(M=4.25, D T=0.92)$.

\section{Diferencias en las percepciones de los riesgos de Internet para personas con y sin discapacidad intelectual}

En cuanto a la comparación entre las valoraciones de los estudiantes de Magisterio de los riesgos de Internet para personas con y sin IDD, los participantes consideran que dichos riesgos son mayores para las personas con IDD que para el resto de la población, observándose diferencias estadísticamente significativas entre las valoraciones globales realizadas sobre dichos grupos $[t(120)=6.027, p=.000, d$ de Cohen $=.422]$. En general, los futuros maestros consideran que las personas con IDD corren mayores riesgos al utilizar Internet que las personas sin IDD.

Cuando se analizan cada uno de los riesgos, se observa que las diferencias estadísticamente significativas se dan en 25 de los 30 ítems estudiados y que en todos los casos se percibe un mayor riesgo de Internet para las personas con IDD que para las personas sin IDD (Tabla 1). Así, por ejemplo, frente al $50 \%$ de los participantes que considera que la población general puede ser acosada a través de Internet, el $72 \%$ opina que esto mismo les puede suceder a las personas con IDD. Los resultados indican, además, diferencias más acusadas $(p<.000)$ en aquellos ítems relacionados con la cibervictimización (e.g. ser acosado, amenazado o acechado online), la exposición a contenidos online inapropiados (e.g. contenido pornográfico ofensivo, drogas, conductas extremistas y antisociales), en aquellos ítems relacionados con el gasto de dinero a través de la Red (e.g. apuestas, compras, gestiones online), así como otras situaciones como pasar demasiado tiempo en las redes sociales, ser víctimas de fraudes o timos, tener dificultad para discriminar la veracidad de la información o facilitar las contraseñas a otras personas. En la mayoría de los casos los tamaños del efecto fueron medianos.

Por otro lado, no se observan diferencias estadísticamente entre ambos grupos $(p>.05)$ en cuestiones relacionadas con un uso excesivo de la red, como hacerse adicto al uso de las redes sociales o pasar menos tiempo que con la familia y amigos, así como en cuestiones relacionadas con la participación activa en determinadas conductas como el acoso a otras personas, crear 0 escribir contenido dañino en Internet y difundir fotos o información personal de otras personas sin su consentimiento. 
Tabla 1. Comparación de las percepciones de los estudiantes de Magisterio de los riesgos de Internet para personas con y sin discapacidad intelectual y del desarrollo

\begin{tabular}{|c|c|c|c|c|c|c|c|}
\hline \multirow[b]{2}{*}{ Riesgos } & \multicolumn{2}{|c|}{$\begin{array}{l}\text { Personas } \\
\text { con IDD }\end{array}$} & \multicolumn{2}{|c|}{$\begin{array}{l}\text { Personas sin } \\
\text { IDD }\end{array}$} & \multirow[b]{2}{*}{$t(120)$} & \multirow[b]{2}{*}{$p$} & \multirow[b]{2}{*}{$\begin{array}{c}d \text { de } \\
\text { Cohen }\end{array}$} \\
\hline & $M$ & $S D$ & $M$ & $S D$ & & & \\
\hline $\begin{array}{l}\text { 1. Ser acosado/-a (e.g. insultos, } \\
\text { vejaciones). }\end{array}$ & 4.64 & 0.61 & 4.24 & 0.85 & -5.868 & .000 & .553 \\
\hline $\begin{array}{l}\text { 2. Tener contacto con personas } \\
\text { desconocidas por ellos/-as. }\end{array}$ & 4.55 & 0.75 & 4.19 & 0.91 & -4.088 & .000 & .382 \\
\hline $\begin{array}{l}\text { 3. Ser expuesto/-a a contenido } \\
\text { pornográfico inapropiado u ofensivo que } \\
\text { no desea ver. }\end{array}$ & 4.50 & 0.74 & 4.17 & 0.95 & -4.276 & .000 & .398 \\
\hline $\begin{array}{l}\text { 4. Ser expuesto/-a a material inapropiado } \\
\text { relacionado con las drogas que no desea } \\
\text { ver. }\end{array}$ & 4.52 & 0.73 & 4.11 & 0.98 & -6.068 & .000 & .576 \\
\hline $\begin{array}{l}\text { 5. Ser expuesto/-a a material que fomente } \\
\text { la conducta antisocial y la intolerancia } \\
\text { (e.g. sexismo, homofobia, agresiones). }\end{array}$ & 4.50 & 0.68 & 4.25 & 0.86 & -3.777 & .000 & .359 \\
\hline $\begin{array}{l}\text { 6. Aportar demasiada información } \\
\text { personal. }\end{array}$ & 4.54 & 0.68 & 4.38 & 0.78 & -2.115 & .036 & .199 \\
\hline $\begin{array}{l}\text { 7. Quedar con alguien en persona que } \\
\text { previamente había conocido en Internet. }\end{array}$ & 4.44 & 0.77 & 4.16 & 0.95 & -3.111 & .002 & .287 \\
\hline 8.Ser amenazado/-a. & 4.55 & 0.70 & 4.20 & 0.90 & -4.796 & .000 & .444 \\
\hline $\begin{array}{l}\text { 9. Limitar o afectar a sus interacciones } \\
\text { cara a cara. }\end{array}$ & 4.46 & 0.69 & 4.17 & 0.88 & -3.541 & .001 & .329 \\
\hline $\begin{array}{l}\text { 10. Afectar a su salud física por pasar } \\
\text { demasiado tiempo en Internet. }\end{array}$ & 4.42 & 0.73 & 4.05 & 0.91 & -4.770 & .000 & .441 \\
\hline $\begin{array}{l}\text { 11. Hacerse adicto/-a al uso de las redes } \\
\text { sociales. }\end{array}$ & 4.44 & 0.69 & 4.34 & 0.82 & -1.382 & .170 & .128 \\
\hline $\begin{array}{l}\text { 12. Pasar menos tiempo trabajando, } \\
\text { aprendiendo o en su desarrollo personal. }\end{array}$ & 4.39 & 0.72 & 4.17 & 0.87 & -2.819 & .006 & .258 \\
\hline 13. Participar en el acoso a otras personas. & 3.93 & 1.12 & 3.88 & 1.08 & -.485 & 628 & .037 \\
\hline $\begin{array}{l}\text { 14. Pasar menos tiempo con la familia y } \\
\text { amistades. }\end{array}$ & 4.21 & 0.87 & 4.17 & 0.86 & -.501 & 617 & .044 \\
\hline $\begin{array}{l}\text { 15. Ser expuesto/-a a contenido violento o } \\
\text { gore que no desea ver. }\end{array}$ & 4.32 & 0.80 & 4.15 & 0.87 & -2.476 & .015 & .222 \\
\hline 16. Ser susceptible de fraudes o timos. & 4.50 & 0.70 & 4.22 & 0.82 & -3.775 & .000 & .347 \\
\hline 17.Ser hostigado/-a o acechado/-a online. & 4.45 & 0.73 & 4.11 & 0.85 & -4.754 & .000 & .428 \\
\hline $\begin{array}{l}\text { 18. Crear o escribir en Internet contenido } \\
\text { dañino. }\end{array}$ & 4.07 & 1.00 & 3.93 & 0.97 & -1.443 & .152 & .140 \\
\hline $\begin{array}{l}\text { 19. Ser acosado/-a por un adulto con una } \\
\text { clara intención sexual (grooming). }\end{array}$ & 4.37 & 0.81 & 4.20 & 0.90 & -2.476 & .015 & .224 \\
\hline $\begin{array}{l}\text { 20. Participar en páginas de juegos o } \\
\text { apuestas online. }\end{array}$ & 4.28 & 0.82 & 3.86 & 0.96 & -4.992 & .000 & .458 \\
\hline $\begin{array}{l}\text { 21. Realizar descargas ilegales y violar los } \\
\text { derechos de autor. }\end{array}$ & 4.26 & 0.83 & 4.09 & 0.96 & -2.057 & .042 & .186 \\
\hline $\begin{array}{l}\text { 22. Que otras personas utilicen } \\
\text { indebidamente sus datos personales. }\end{array}$ & 4.50 & 0.73 & 4.30 & 0.81 & -2.832 & .005 & .261 \\
\hline $\begin{array}{l}\text { 23. Que alguien acceda ilegalmente a sus } \\
\text { cuentas y perfiles en Internet. }\end{array}$ & 4.44 & 0.74 & 4.20 & 0.93 & -3.501 & .001 & .329 \\
\hline $\begin{array}{l}\text { 24. Descargar involuntariamente spyware } \\
\text { o malware en su ordenador (e.g. virus). }\end{array}$ & 4.42 & 0.75 & 4.26 & 0.89 & -2.040 & .044 & .191 \\
\hline 25. Difundir fotos suyas. & 4.36 & 0.80 & 4.19 & 0.89 & -2.018 & .046 & .182 \\
\hline $\begin{array}{l}\text { 26. Difundir fotos o información personal } \\
\text { de otras personas sin su autorización. }\end{array}$ & 4.31 & 0.81 & 4.25 & 0.92 & -.818 & .415 & .078 \\
\hline $\begin{array}{l}\text { 27. Tener dificultad para discriminar la } \\
\text { veracidad de la información de Internet. }\end{array}$ & 4.56 & 0.66 & 4.17 & 0.88 & -4.710 & .000 & .441 \\
\hline $\begin{array}{l}\text { 28. Facilitar su contraseña a otras } \\
\text { personas. }\end{array}$ & 4.35 & 0.85 & 3.97 & 1.07 & -4.244 & .000 & .394 \\
\hline $\begin{array}{l}\text { 29. Realizar compras online o gestiones } \\
\text { donde se requieran cuentas bancarias. }\end{array}$ & 4.26 & 0.86 & 3.88 & 0.96 & -4.086 & .000 & .383 \\
\hline 30. Desarrollar una adicción a Internet & 4.37 & 0.72 & 4.15 & 0.91 & -2.988 & .003 & .276 \\
\hline
\end{tabular}




\section{DISCUSIÓN}

El objetivo principal de este estudio era conocer las percepciones que tienen los futuros maestros acerca de los riesgos de Internet. Concretamente, se pretendía analizar si dichas percepciones diferían en función de si el uso de Internet lo realizan personas con IDD o personas sin IDD. LoS principales resultados muestran una alta percepción de riesgo del uso de Internet para ambos colectivos, si bien dichas percepciones son mayores para el grupo de personas con IDD. La diferencias fueron estadísticamente significativas en prácticamente todos los riesgos incluidos en el cuestionario (25 de 30 ítems) y todos en el mismo sentido, es decir, los estudiantes de Magisterio consideran que las personas con IDD corren un mayor riesgo al utilizar Internet que las personas que no tienen ninguna discapacidad. Estos resultados coinciden plenamente con los obtenidos en el estudio de Chadwick et al. (2017) en los que también se encontró este patrón, tanto para el total de la escala como para cada uno de los riesgos analizados. Los resultados de ambos estudios muestran la tendencia que hay en la población general a percibir a las personas con IDD como más vulnerables a las conductas inapropiadas de los otros, en este caso particular en un entorno virtual. De hecho, si se estudian con más detalle los riesgos, se observa que los estudiantes de Magisterio muestran una mayor preocupación por aquellos ítems que implican cibervictimización (e.g. acoso, amenazas, hostigamiento) y cierto grado de inocencia por parte de las personas con IDD, como quedar con desconocidos, aportar demasiada información personal, tener dificultad para discriminar la veracidad de la información, ser víctima de fraudes y timos o gastar dinero online. Sin embargo, aquellos aspectos relacionados con una implicación activa de la persona con IDD en el ciberacoso, no son considerados como un peligro.

La forma en como las personas entienden Ia IDD va a determinar el grado en que va a facilitar el acceso de estas personas al mundo digital. Si se entiende que Internet va a suponer un mayor riesgo para las personas con IDD en comparación al resto de los ciudadanos, la posibilidades de favorecer su inclusión digital van a ser escasas debido a una tendencia a protegerlos de los potenciales peligros que se dan en la Red (Chadwick \& Wesson, 2016; Lussier-Desrochers et al., 2017). Aun así, ciertos estudios indican que, a pesar de las preocupaciones de los cuidadores, los beneficios de Internet compensan a los posibles riesgos que se derivan de él (Molin, Sorbring, \& LöfgrenMartenson, 2015) por lo que hay que promocionar su uso. Por otro lado, la perspectiva de la 'gestión positiva de los riesgos' es un área poco explorada en el ámbito de la discapacidad intelectual y el uso de Internet y que, sin embargo, puede ayudar a favorecer el uso responsable de esta herramienta, sintiendo, tanto los propios usuarios como sus cuidadores, que tienen controlados los riesgos. Desde este enfoque se defiende la idea de que las personas con IDD deberían tomar sus propias decisiones acerca de sus vidas y de cómo vivirla (Seale, 2014; Seale \& Chadwick, 2017). Más que evitarse los riesgos en Internet se debe dotar a los usuarios con las herramientas que le permitan reconocer y gestionar dichos riesgos y ganar, de esta forma, un mayor control sobre su vida y bienestar. En definitiva, se trata de que profesores, profesionales y personas con IDD trabajen para acordar las acciones que deben seguir teniendo en cuenta los potenciales resultados positivos de sus acciones, así como lo negativos (McConkey \& Smyth, 2003).

Las respuestas dadas por los estudiantes de Magisterio nos llevan a pensar que todavía existen ciertos prejuicios acerca de la capacidad de las personas con IDD de llevar una vida similar a la del resto de la población. Desde la formación inicial de los docentes se deberían trabajar cuestiones como las actitudes y las percepciones que tienen hacia las personas con discapacidad y dotarles de las estrategias que sean necesarias para que en su futuro laboral más inmediato faciliten la inclusión de las personas con discapacidad en todos los ámbitos de la vida, incluida la digital. Además, un mayor conocimiento de los beneficios, de los riesgos y de la seguridad en Internet, dotará a los 
profesores de las herramientas para favorecer el acceso online de las personas con IDD de una manera responsable y segura.

El estudio presenta algunas limitaciones que deben ser tenidas en cuenta. En primer lugar, los participantes en el estudio son todo estudiantes de una única facultad por lo que no se pueden generalizar los resultados a otros contextos. Sería necesario ampliar la muestra y contar con la participación de estudiantes de otras universidades. En segundo lugar, una perspectiva más completa del estudio se obtendría si se contara también con la participación de profesores en activo. En este caso se podrían analizar las posibles diferencias con respecto a los estudiantes de Magisterio, ya que su experiencia puede haber derivado en un cambio de actitudes y de las percepciones de riesgo. En tercer lugar, el conocimiento que tengan los participantes sobre Internet puede afectar en sus respuestas, percibiendo menores riesgos aquellos estudiantes que utilizan con mayor frecuencia Internet o en más ámbitos de su vida (e.g. compras, transacciones, formación, ocio). Futuras investigaciones deberían tener en cuenta este factor. Por último, la perspectiva de los propios usuarios, es decir, las personas con IDD aportarían mayor valor al estudio. Hay que tener en cuenta que el presente estudio hace referencia únicamente a los posibles riesgos que pueden darse en Internet, pero no entra a valorar los riesgos reales que han sufrido los usuarios (Chadwick et al., 2017). Estas y otras cuestiones ayudarían a conocer más sobre un tema que apenas se ha trabajado y que resulta de gran importancia para el desarrollo personal y social de las personas con IDD a través de su inclusión digital.

\section{CONCLUSIONES}

El presente estudio pone de manifiesto las percepciones que tienen los estudiantes de Magisterio sobre el uso de Internet, especialmente para las personas con IDD. Se ha visto cómo consideran que los riesgos de esta herramienta son mayores para estas personas que para las personas sin discapacidad lo que puede perpetuar la brecha digital. Se observa también la percepción de vulnerabilidad de las personas con IDD por lo que resulta necesario poner en marcha programas que favorezcan la adquisición de estrategias que permitan la gestión de los riesgos y maximicen los beneficios de Internet. Para ello, resulta necesario seguir investigando con el objeto de obtener una mayor evidencia científica en este campo que permita la participación plena de todas las personas en el mundo digital, independientemente de sus características individuales.

\section{REFERENCIAS}

Caton, S., \& Chapman, M. (2016). The use of social media and people with intellectual disability: A systematic review and thematic analysis. Journal of Intellectual \& Developmental Disability, 41(2), 125-139. doi: 10.3109/13668250.2016.1153052

Chadwick, D. D., Fullwood, C., \& Wesson, C. J. (2013). Intellectual disability, identity, and the internet. En R. Luppicini (Ed.), Handbook of research on technoself: Identity in a technological society (pp. 229-254). Hershey, PA: Information Science

Chadwick, D. D., Quinn, S., \& Fullwood, C. (2017). Perceptions of the risks and benefits of internet access and use by people with intellectual disabilities. British Journal of Learning Disabilities, 45(1), 21-31. doi: 10.1111/bld.12170

Chadwick, D. D., \& Wesson, C. (2016). Digital inclusion and disability. En A. Atrill, \& C. Fullwood (Eds.), Applied cyberpsychology (pp. 1-23). Houndmills, UK: Palgrave Macmillan. doi: 10.1057/9781137517036_1

Chiner, E., Gómez-Puerta, M., \& Cardona-Moltó, M. C. (2017a). Internet and people with intellectual disability: An approach to caregivers' concerns, prevention strategies and training needs. 
[Internet y personas con discapacidad intelectual: una aproximación a las preocupaciones, estrategias de prevención y necesidades de formación de los cuidadores] Journal of New Approaches in Educational Research, 6(2), 153-158. doi: 10.7821/naer.2017.7.243

Chiner, E., Gómez-Puerta, M., \& Cardona-Moltó, M. C. (2017b). Internet use, risks and online behaviour: The view of internet users with intellectual disabilities and their caregivers. British Journal of Learning Disabilities, 45(3), 190-197. doi: 10.1111/bld.12192

Didden, R., Scholte, R. H. J., Korzilius, H., De Moor, Jan M H, Vermeulen, A., O’Reilly, M., . . . Lancioni, G. E. (2009). Cyberbullying among students with intellectual and developmental disability in special education settings. Developmental Neurorehabilitation, 12(3), 146-151. doi: $10.1080 / 17518420902971356$

Gutiérrez, P., \& Martorell, A. (2011). People with intellectual disability and ICTs. [Las personas con discapacidad intelectual antes las TIC] Comunicar, 18(36), 173-180. doi: 10.3916/C36-2011-0309

Jenaro, C., Flores, N., Vega, V., Cruz, M., Pérez, M. C., \& Torres, V. A. (2018). Cyberbullying among adults with intellectual disabilities: Some preliminary data. Research in Developmental Disabilities, 72, 265-274. doi: 10.1016/j.ridd.2017.12.006

Jones, L. M., Mitchell, K. J., \& Finkelhor, D. (2012). Trends in youth internet victimization: Findings from three youth internet safety surveys 2000-2010. Journal of Adolescent Health, 50(2), 179186. doi: 10.1016/j.jadohealth.2011.09.015

Livingstone, S., \& Haddon, L. (2009). EU kids online: Final report. LSE, London, UK: EU Kids Online. (EC Safer Internet Plus Programme Deliverable D6.5).

Livingstone, S., Haddon, L., Görzig, A., \& Ólafsson, K. (2011). Risks and safety on the internet: the perspective of european children. full findings. LSE, London, UK: EU Kids Online.

Löfgren-Mårtenson, L. (2008). Love in cyberspace. Swedish young people with intellectual disabilities and the internet. Scandinavian Journal of Disability Research, 10(2), 125-138. doi: 10.1080/15017410701758005

Lussier-Desrochers, D., Normand, C. L., Romero-Torres, A., Lachapelle, Y., Godin-Tremblay, V., Dupont, M. È, ... Bilodeau, P. (2017). Bridging the digital divide for people with intellectual disability. Cyberpsychology: Journal of Psychosocial Research on Cyberspace, 11(1), article 1. doi: 10.5817/CP2017-1-1

McConkey, R., \& Smyth, M. (2003). Parental perceptions of risks with older teenagers who have severe learning difficulties contrasted with the young people's views and experiences. Children \& Society, 17(1), 18-31. doi: 10.1002/chi.725

Molin, M., Sorbring, E., \& Löfgren-Martenson, L. (2015). Teachers' and parents' views on the internet and social media usage by pupils with intellectual disabilities. Journal of Intellectual Disabilities, 19(1), 22-33. doi: 10.1177/1744629514563558

Normand, C. L., Lussier-Desrochers, D., Fecteau, S., Godin-Tremblay, V., Dupont, M., Roux, J., \& Romero, A. (2016). A conceptual model of factors leading to the digital exclusion of people with neurodevelopmental disorders. Annual Review of Cybertherapy and Telemedicine, 14, 23-29.

Palmer, S. B., Wehmeyer, M. L., Davies, D. K., \& Stock, S. E. (2012). Family members' reports of the technology use of family members with intellectual and developmental disabilities. Journal of Intellectual Disability Research, 56(4), 402-414. doi: 10.1111/j.1365-2788.2011.01489.x

Schalock, R. L., Borthwick-Duffy, S., Bradley, V. J., Buntinx, W. H. E., Coulter, D. L., Craig, E. M., . . . Yeager, M. H. (2010). Intellectual disability: Definition, classification, and systems of supports (11th ed.). Washington, DC. USA: American Association on Intellectual and Developmental Disabilities. 
Seale, J. (2014). The role of supporters in facilitating the use of technologies by adolescents and adults with learning disabilities: A place for positive risk-taking. European Journal of Special Needs Education, 29(2), 220-236. //dx.doi.org/10.1080/08856257.2014.906980

Seale, J., \& Chadwick, D. (2017). How does risk mediate the ability of adolescents and adults with intellectual and developmental disabilities to live a normal life by using the internet? Cyberpsychology: Journal of Psychosocial Research on Cyberspace, 11(1), article 2. doi: 10.5817/CP2017-1-2

Wells, M., \& Mitchell, K. J. (2014). Patterns of internet use and risk of online victimization for youth with and without disabilities. The Journal of Special Education, 48(3), 204-213. doi: $10.1177 / 0022466913479141$

World Health Organization. (2011). World report on disability. Malta: Author. 